\title{
Transmission and charge state distribution of carbon ions emerging from nitrogen gas target in a tandem accelerator: Impact of stripper gas pressure
}

\author{
Mihir Sarkar, ${ }^{1}$ Neeraj Shukla, ${ }^{1}$ Nobin Banerji, ${ }^{1}$ and Y. N. Mohapatra ${ }^{1,2,3, *}$ \\ ${ }^{1}$ Department of Physics, Indian Institute of Technology Kanpur, Kanpur 208016, India \\ ${ }^{2}$ Materials Science Programme, Indian Institute of Technology Kanpur, Kanpur 208016, India \\ ${ }^{3}$ Samtel Center for Display Technologies, Indian Institute of Technology Kanpur, Kanpur 208016, India
}

(Received 5 June 2012; published 17 October 2012)

\begin{abstract}
In a tandem accelerator the knowledge of distribution of charge states as well as the overall transmission of incident ions is of great importance for optimized use of the accelerator. We have studied the effect of the stripper gas pressure on the charge state distribution and the transmission of incident carbon ions $\left({ }^{12} \mathrm{C}^{1-}\right)$ stripped in $\mathrm{N}_{2}$ gas target in a tandem accelerator in the energy range of $0.2-1.7 \mathrm{MeV}$. The observed charge state fractions of the outgoing beam show transition from nonequilibrium to equilibrium distribution as the stripper gas pressure is varied. The experimentally measured average charge values at different stripper gas pressures are compared with an empirical model for the equilibrium average charge. Apart from the charge state distribution, the stripper gas pressure is found to have a more interesting effect in the transmission of incident carbon ions $\left({ }^{12} \mathrm{C}^{1-}\right)$, which displays a maximum with pressure in the nonequilibrium region. The observed transmission behavior is explained qualitatively.
\end{abstract}

DOI: 10.1103/PhysRevSTAB.15.100101

PACS numbers: 29.20.Ej, 29.27.Ac, 34.50.Fa

\section{INTRODUCTION}

Low energy tandem accelerators are used for extraction and acceleration of various ion species for material science research. The energetic ions are used for implantation, doping, surface modification, and many more applications $[1,2]$. In a tandem accelerator the negatively charged ions are extracted from the source and accelerated to high velocity by the large positive potential at the terminal. The high velocity negative ion beam then passes through the stripper medium present at the center of the acceleration tube. The stripper media may be a gas or thin solid foil. In the stripper medium the negative ions lose some electrons and become positive. These positive ions are further accelerated by the difference between positive potential at the terminal and the ground potential at the end of the high energy acceleration column. The ion beam which enters the high energy acceleration column after passing through the stripper medium consists of different charge states and these are accelerated to different energies while emerging out of the high energy end of the acceleration column. In the stripper medium the high speed ions lose and capture electrons in collisions with stationary target atoms while they pass through the medium. As a result, the average charge of the ion beam fluctuates. After a sufficient number of collisions, equilibrium is reached and the average charge and the distribution of charge state fractions do not change

\footnotetext{
*ynm@iitk.ac.in
}

Published by the American Physical Society under the terms of the Creative Commons Attribution 3.0 License. Further distribution of this work must maintain attribution to the author(s) and the published article's title, journal citation, and DOI. with further increment of the target thickness. The incident ions should pass through a minimum target thickness to have sufficient collisions to reach equilibrium charge state distribution. The equilibrium average charge depends primarily on the incident ion velocity (energy) and nuclear charge of the ion. There exist theoretical models for prediction of the equilibrium average charge state $[3,4]$. The inadequacy of a single theory, to predict the average charge of ions of all masses and energy range has led to different semiempirical formulas [5]. However, the charge state distribution of the beam emerging from the high energy acceleration column may be slightly different than that of the beam just after the stripper medium due to an unequal amount of elastic scattering of ions of different charge states from the residual gas molecules in the high energy acceleration column. Thus, it would be interesting to check the average charge state in the beam coming out of the high energy acceleration column in a typical tandem accelerator and compare with equilibrium average charge predicted by a semiempirical formula for that stripper target and beam. Also the study of transmission of incident ions, which depends in a complex manner on the incident ion energy and target medium thickness, is important for optimization of the accelerator.

In this paper, we specifically choose to study the important case of carbon beam with focus on charge state distribution and the transmission in a standard tandem accelerator. For carbon atoms, previous studies have been limited to the energy range above $3 \mathrm{MeV}$ just after it passes through solid foil targets [6,7], while for stripping in a gaseous media it has been reported in the energy range above $1.5 \mathrm{MeV}$ and below $0.6 \mathrm{MeV}$ [8-10]. The variation in transmission with stripper gas pressure has been reported 
by Jacob et al. only at two different sub-MeV energies [10]. In this work, we have studied charge state distribution and transmission of carbon $\left({ }^{12} \mathrm{C}\right.$ ) ions (at the high energy end of acceleration column) stripped in $\mathrm{N}_{2}$ gas in a tandem accelerator, with specific focus on effects of stripper gas pressure. The energy of the incident ions was varied in the range of $0.2-1.7 \mathrm{MeV}$, which is useful for many material science studies with such ion beams. We have critically compared our experimental average charge $\left(q_{\text {avg }}\right)$ at different stripper gas pressures with equilibrium average charge predicted by an empirical formula recently developed by Schiwietz et al. [11]. The effects of stripper gas pressure on transmission $(N)$ in the energy range of the present study are analyzed. We show that the stripper gas pressure variation can lead to transition from nonequilibrium to equilibrium target thickness beyond a threshold of target thickness. The distribution of fractions of charge states $\left(F_{q}\right)$ at nonequilibrium and equilibrium target thicknesses (due to variation in pressure) are also reported and discussed.

\section{EXPERIMENTAL PROCEDURE}

The extraction and acceleration of the incident carbon ions $\left({ }^{12} \mathrm{C}^{1-}\right)$ and their subsequent stripping were carried out in a High Voltage Engineering Europa made 1.7 MV Tandetron $^{\mathrm{TM}}$ accelerator, which is a tandem type of accelerator. The negative carbon ions are produced in a source of negative ion by cesium sputtering (SNICS) source, and extracted out from the source chamber by an applied voltage of $30 \mathrm{kV}$. For mass and charge selection $\left({ }^{12} \mathrm{C}^{1-}\right)$, the extracted negative ion beam is passed through a $90^{\circ}$ analyzing magnet [see Fig. 1]. This monochromatic beam then enters the acceleration tube. Here the ions go through the two stages of acceleration. The acceleration tube is 4 meters long with the stripper canal at the central portion. This stripper canal is of $86 \mathrm{~cm}$ length and $13 \mathrm{~mm}$ diameter. At the center of the canal, nitrogen stripper gas is fed through a small inlet hole on the wall of the canal and pumped out through an outlet hole on the opposite wall. The different stripper gas pressures in the canal are achieved by adjusting the needle valve at the inlet. The beam which comes out of the high energy acceleration tube consists of neutrals and various positive charge states. Energy of an outgoing particle having charge state $q$ is $e \times(1+q) \times V$, where $V$ is the voltage applied at the terminal and $e$ is the electronic charge. The different charge states of the outgoing beam are separated by a high energy switching magnet situated after the acceleration tube and the currents for different positive charge states are measured at an electron suppressed Faraday cup (FC2) fixed at the $45^{\circ}$ port of the switching magnet as shown in Fig. 1. The current of the incident ${ }^{12} \mathrm{C}^{1-}$ ions is measured at another Faraday cup (FC1) situated after the $90^{\circ}$ analyzing magnet. FC1 is a retractable Faraday cup placed before the accelerator and is used to measure the low energy incident beam current. A bias voltage of $-60 \mathrm{~V}$ is provided to $\mathrm{FC} 1$ to suppress the secondary electrons and measure the incident current accurately. For any fixed incident ion energy, the switching magnet and the quadrupole are adjusted for focusing and measuring the current of a charge state $\left(I_{q}\right)$ at FC2. Then immediately the current of the incident ${ }^{12} \mathrm{C}^{1-}$ beam at that time $\left(I_{q}^{-}\right)$is also measured using the retractable Faraday cup (FC1). The incident current is measured for every charge state and energy of the incident ion beam. During measurement of two consecutive charge states, the incident current changes negligibly (by less than 1\%). In normal operation with a stable source, during the course of measurement in the entire range of energy, the incident current changes by less than 5\%. However, the fluctuation of the incident current (by any amount) does not affect the calculation of $q_{\text {avg }}$ or $N$. The incident currents $\left(I_{q}^{-}\right)$serve as normalization values to take care of any small fluctuations of the incident beam current during the measurements. The average charge of the outgoing beam is defined as $q_{\text {avg }}=$ $\sum q F_{q}$. The fraction of charge state $q\left(F_{q}\right)$ in the outgoing beam is calculated by the formula $F_{q}=N_{q} / \sum N_{q}$, where $N_{q}=I_{q} / q I_{q}^{-}$is the normalized number of particles, having charge state $q$, in the outgoing beam. The normalized transmission of the incident ${ }^{12} \mathrm{C}^{-}$ions is given by $N=\sum N_{q}$. The charge state fractions estimated this way were fitted

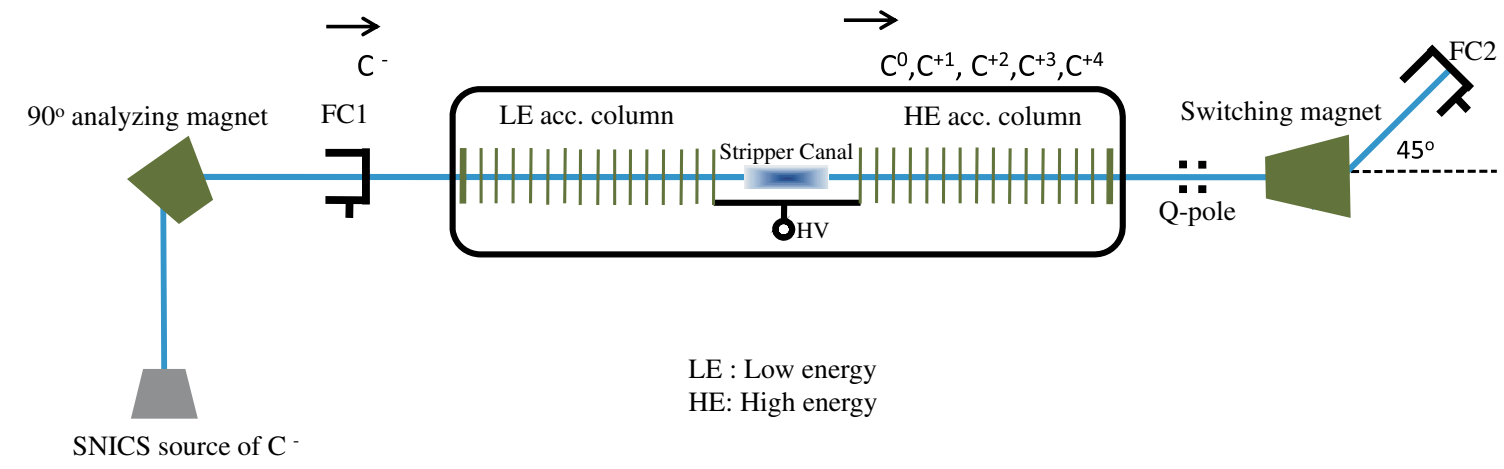

FIG. 1. The schematic diagram of the experimental setup. FC1 and FC2 are the two Faraday cups used to measure the currents of the incident and the outgoing beam, respectively. 
with Gaussian curves of widths $d=\left[\sum_{q}\left(q-q_{\text {avg }}\right)^{2} \times F_{q}\right]^{1 / 2}$, centered at $q_{\text {avg }}$. For a fixed stripper gas pressure, we varied the terminal voltage of the accelerator from $0.2-1.7 \mathrm{MV}$ in steps of $0.1 \mathrm{MV}$ and carried out all the above-mentioned current measurements. The procedure was repeated for different stripper gas pressures.

\section{RESULTS AND DISCUSSION}

\section{A. Average charge state and equilibrium target thickness}

Theoretical and semiempirical formulas for prediction of equilibrium average charge state of energetic ions stripped in solid and gaseous media were developed by Bohr, Betz, Dmitriev, and Nikolaev at different stages $[5,12,13]$. Schiwietz et al. has developed a comparatively new improved empirical formula for equilibrium average charges of ions stripped in gaseous media [11].

We have compared the average charges measured in our experiments with the equilibrium average charge values predicted by Schiwietz's formula. The Schiwietz's formula for equilibrium average charge is a multiparameter leastsquares fit to 550 experimental data points for gas targets. The average equilibrium charge is given as

$$
q_{\mathrm{avg}}=Z_{p} \frac{376 x+x^{6}}{1428-1206 x^{1 / 2}+690 x+x^{6}},
$$

where

$$
x=\left[\frac{v_{p}}{v_{0}} Z_{p}^{-0.52} Z_{t}^{0.03-0.017 z_{p}^{-0.52}\left(v_{p} / v_{0}\right)}\right]^{1+\left(0.4 / Z_{p}\right)} .
$$

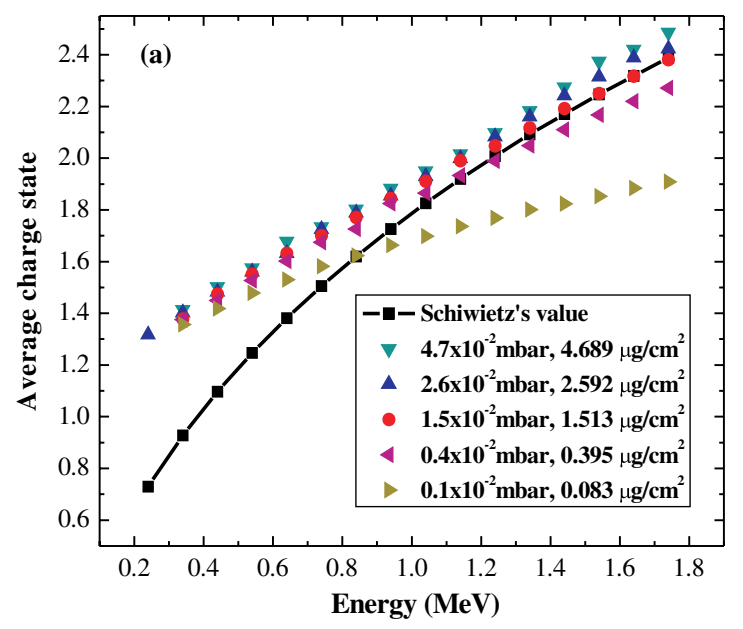

$Z_{p}$ and $v_{p}$ are the projectile atomic number and velocity, respectively. $Z_{t}$ is the target atomic number and $v_{0}$ is the Bohr velocity $\left(2.19 \times 10^{6} \mathrm{~m} / \mathrm{s}\right)$. This is a single formula for prediction of equilibrium average charge of all ions in gas targets. The experimental data points used in the leastsquares fit included ions having velocity higher than a threshold velocity $v_{p} / v_{0}=0.7$, for $Z_{p}>2$. In the energy range of our experiments $v_{p} / v_{0}>1$, thus it is suitable to compare our data with the formula. The relative uncertainty in prediction of the equilibrium average charge by this formula is $\Delta q_{\text {avg }} / Z_{p}=2.6 \%$ [11]. Figure 2(a) shows the experimentally obtained average charge data points for carbon ions in the incident ion energy range $0.2-1.7 \mathrm{MeV}$ for different stripper gas pressures along with the empirical values of equilibrium average charges estimated using Eq. (1). It is observed that for energies above $1 \mathrm{MeV}$ the average charge data at $1.5 \times 10^{-2}$ mbar of stripper canal pressure matches pretty well with the Schiwietz's prediction, whereas there is always a mismatch between the experimental and empirical value for incident ion energies below $1 \mathrm{MeV}$. The mismatch in the low energy side is discussed later in the manuscript.

Each stripper gas pressure corresponds to an equivalent target thickness. A change of pressure in the stripper canal manifests through the change of pressure at the high energy (HE) terminal of the accelerator tube [see Fig. 1]. We measure the pressure at the high energy (HE) terminal of the acceleration tube and calculate the average pressure in the stripper canal by using an empirical formula provided by the manufacturer of the accelerator. The pressure at the center of the stripper canal $\left(P_{\text {center }}\right)$ in terms of pressure at the $\mathrm{HE}$

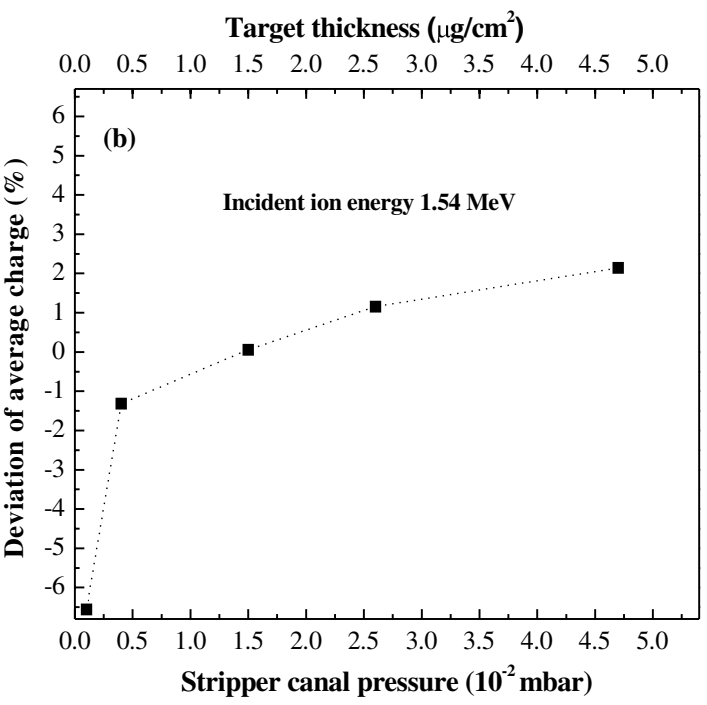

FIG. 2. (a) Variation of average charge of the outgoing beam with incident ion energy measured at different stripper gas pressures. The target thicknesses were calculated for $\mathrm{N}_{2}$ gas, taking into account the average pressure in the stripper canal and its length. The connected data points represent the empirical values of equilibrium average charge predicted by Schiwietz's formula [11]. (b) Variation of deviation of experimental average charge $\left(q_{\mathrm{avg}}^{\mathrm{exp}}-q_{\mathrm{avg}}^{\mathrm{emp}}\right) / Z_{p}$ with stripper gas pressure, at $1.54 \mathrm{MeV}$ of incident ion energy. 
terminal $\left(P_{\mathrm{HE}}\right)$ is given by $P_{\text {center }}=A \times \ln \left[\left(P_{\mathrm{HE}}-B\right) / C\right]$, where $A=-0.074, B=1.371 \times 10^{-5} \mathrm{mbar}$, and $C=$ $-1.359 \times 10^{-5} \mathrm{mbar}$. The pressure at the end of the stripper canal is about 100 times lower than the pressure at the center. We calculate the average pressure over the stripper canal assuming a linear pressure drop from the center of the stripper canal to the ends. The target thickness (in $\mu \mathrm{g} / \mathrm{cm}^{2}$ ) is estimated for each stripper canal pressure considering the $86 \mathrm{~cm}$ long stripper canal. The pressure measurement has uncertainty due to unavoidable fluctuation in measured pressure at the HE terminal during normal operation. The fluctuation observed in the $P_{\mathrm{HE}}$ is $\Delta P_{\mathrm{HE}} \sim 0.3 \times 10^{-6}$ mbar. We have estimated the propagation of this error into the pressure value at the center of the stripper canal $\left(P_{\text {center }}\right)$, using the formula for pressure at the center. Subsequently, we have estimated the uncertainty in average pressure in the stripper canal, which turns out to be $\sim 8 \%$. This is equal to the uncertainty in target thickness as well. The relative deviation is defined as $\left(q_{\mathrm{avg}}^{\mathrm{exp}}-q_{\mathrm{avg}}^{\mathrm{emp}}\right) / Z_{p}$, where $q_{\mathrm{avg}}^{\exp }$ is the experimentally found average charge and $q_{\text {avg }}^{\text {emp }}$ is the equilibrium average predicted by Schiwietz's formula [Eq. (1)]. Figure 2(b) shows the variation of deviation of average charge with stripper gas pressure at incident ion energy $1.54 \mathrm{MeV}$. One can compare the $\left(q_{\mathrm{avg}}^{\mathrm{exp}}-q_{\mathrm{avg}}^{\mathrm{emp}}\right) / Z_{p}$ with $\Delta q_{\text {avg }} / Z_{p}(2.6 \%)$, the later is the relative uncertainty in prediction of the equilibrium average charge by Eq. (1). For stripper gas pressure of $0.1 \times 10^{-2}$ mbar (target thickness $0.083 \mu \mathrm{g} / \mathrm{cm}^{2}$ ) the deviation is $6.55 \%$ on the lower side, which is quite large compared to the $2.6 \%$. We will see in the next section that this stripper gas pressure leads to a nonequilibrium target thickness. For all the other stripper gas pressures, the deviation $\left(q_{\mathrm{avg}}^{\mathrm{exp}}-q_{\mathrm{avg}}^{\mathrm{emp}}\right) / Z_{p}$ is within $2.6 \%$ and the best match is obtained at a stripper gas pressure of $1.5 \times 10^{-2}$ mbar. Note that there is a gradual increase in average charge as the stripper gas pressure is increased.

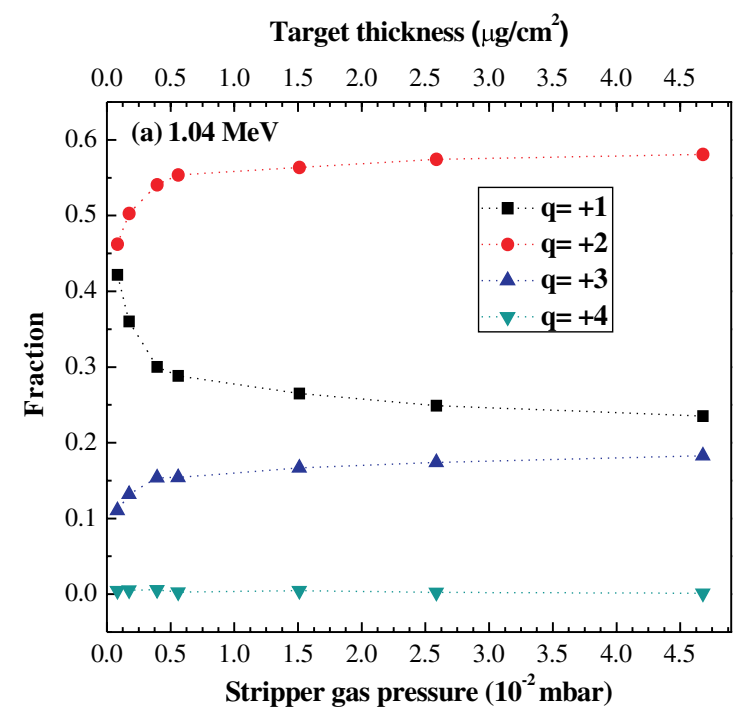

We have estimated the variation of charge state fractions with stripper gas pressures at two different incident ion energies. Figure 3 shows the same at energies 1.04 and $1.44 \mathrm{MeV}$. At both energies the charge state fraction tends towards the equilibrium after $0.6 \times 10^{-2}$ mbar stripper gas pressure, which corresponds to a target thickness of $\sim 0.6 \mu \mathrm{g} / \mathrm{cm}^{2}$. For $2.4 \mathrm{MeV}$ incident carbon ions in $\mathrm{N}_{2}$ stripper gas, we infer from literature [8] that $0.63 \mu \mathrm{g} / \mathrm{cm}^{2}$ target thickness gives equilibrium charge state fractions. As the incident ion energy is less in our experiment, equilibrium is expected at lower target thickness. Figure 3 shows that the target thicknesses beyond $0.6 \mu \mathrm{g} / \mathrm{cm}^{2}$ may be considered to be in the equilibrium range for the energy range of present experiment. In both cases the charge states $q=+2$ and $q=+3$ initially rise and then almost equilibrate, while the charge state $q=+1$ initially reduces and then equilibrates. The equilibrium fraction for $q=+3$ is higher at $1.44 \mathrm{MeV}$ compared to the same at $1.04 \mathrm{MeV}$, which merely justifies the higher $q_{\text {avg }}$ at $1.44 \mathrm{MeV}$. Figure 3 clearly shows that the charge state fractions at stripper gas pressures $0.1 \times 10^{-2}$ and $0.4 \times 10^{-2}$ mbar are certainly nonequilibrium values and, hence, the lower values of $q_{\text {avg }}$ at those pressures are understandable. At stripper gas pressure of $1.5 \times$ $10^{-2}$ mbar (target thickness $1.5 \mu \mathrm{g} / \mathrm{cm}^{2}$ ), the experimentally obtained value of average charge for $1.54 \mathrm{MeV}^{12} \mathrm{C}^{1-}$ is 2.19. Kiisk et al. reported that the average equilibrium charge of $1.5 \mathrm{MeV}^{13} \mathrm{C}^{1-}$ ions stripped in $\mathrm{N}_{2}$ stripper gas is 2.17 [8]. The comparison between the average charge value measured in this experiment and the same reported in the literature shows good agreement. For stripper canal pressure above $1.5 \times 10^{-2}$ mbar, average charge increases further by small amounts on increasing stripper gas pressure. A change in pressure of gas inside the stripper canal will cause a change in residual gas pressure in the high

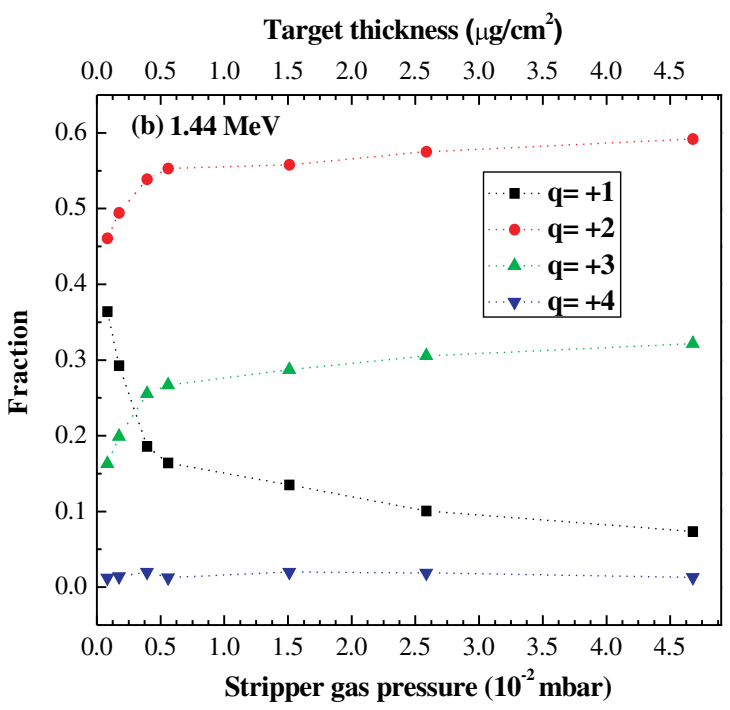

FIG. 3. Variation of different charge state fractions with stripper gas pressure: (a) incident ion energy $1.04 \mathrm{MeV}$, (b) incident ion energy $1.44 \mathrm{MeV}$. 
energy (HE) acceleration column. We have seen that the change in mean square scattering angle $\Delta\left\langle\theta^{2}\right\rangle$ due to multiple scattering of an ion passing through a gaseous medium varies as $1 / v_{p}^{4}$ [14]. Hence, the different charge states produced in the stripper canal will suffer elastic scattering differently in the residual gas of the HE acceleration column of the tandem accelerator. This can cause a slow change in the charge state fractions $\left(F_{q}\right)$ after the equilibrium thickness, which is visible in Fig. 3. The lower charge states will suffer larger scattering due to the residual gas in the HE column. The change in fraction of charge state +1 is highest in Fig. 3. Thus, the charge state distribution just after the stripping is not exactly the same as the distribution in the outgoing beam in a tandem accelerator. The larger scattering loss of the fraction having charge state +1 may cause a gradual increase in $q_{\text {avg }}$ with increment in stripper canal pressure [see Fig. 2(a)]. At very high density of the target medium, the mean charge value goes above the equilibrium value because of the residual excitation of the ions during multiple collisions $[12,15]$. In the present case, for $1.54 \mathrm{MeV}$ incident ions, as the stripper gas pressure increases from $1.5 \times 10^{-2} \mathrm{mbar}$ to $4.7 \times 10^{-2} \mathrm{mbar}$, the experimental average charge increases by $5.8 \%$. Considering the mean-free path of the stripper gas molecules, we estimated the time duration between two collisions at the highest stripper gas pressure at room temperature to be $\sim 10^{-9} \mathrm{sec}$. The lifetime of excited states of the higher charge states of ${ }^{12} \mathrm{C}$ ions might be comparable with the duration between two consecutive collisions. However, without the estimate of lifetime of excited states of ${ }^{12} \mathrm{C}$ ions, this increment in average charges cannot be conclusively attributed to a density effect.

\section{B. Transmission of ions}

The pressure at the stripper canal has remarkable impact on transmission $(\boldsymbol{N})$ of incident ions over the energy range of our experiment. During normal operation the pressure in the beam line as well as in the acceleration tube is of the order of $10^{-6} \mathrm{mbar}$, which ensures a very low number of charge changing collisions in the passage except in the stripper canal. Thus, the beam that enters the stripper canal consists mostly of negative ions and almost all the charge changing collisions occur in the stripper canal, where the target density is made high by introducing the stripper gas. The transmission $(N)$ is estimated by adding up the normalized fractions $\left(N_{q}\right)$ of incident ${ }^{12} \mathrm{C}^{1-}$ beam getting transmitted with different positive charge states. Figure 4(a) shows the variation of transmission with incident ${ }^{12} \mathrm{C}^{1-}$ ion energy at different stripper gas pressures. Another aspect of transmission is how the transmission varies with stripper gas density for fixed incident ion energy. We have estimated the total change in mean square scattering (elastic) angle $\Delta\left\langle\theta^{2}\right\rangle$ of the incident ion during its journey through the stripper canal [14]. Figure 4(b) shows the variation in the overall ion transmission, and $\Delta\left\langle\theta^{2}\right\rangle$ with stripper canal pressure, at $1.04 \mathrm{MeV}$. Here the estimation of $\Delta\left\langle\theta^{2}\right\rangle$ is done by considering only the multiple scattering inside the stripper canal, where the energy remains the same for all the charge states. We can see that as the stripper gas pressure increases from $0.1 \times 10^{-2}$ mbar to $0.4 \times 10^{-2}$ mbar the transmission as well as $\Delta\left\langle\theta^{2}\right\rangle$ increases (for incoming ion energy $1 \mathrm{MeV}$ ). In this range although the multiple scattering increases with increment in target thickness, the increment in charge changing collisions with increasing target thickness
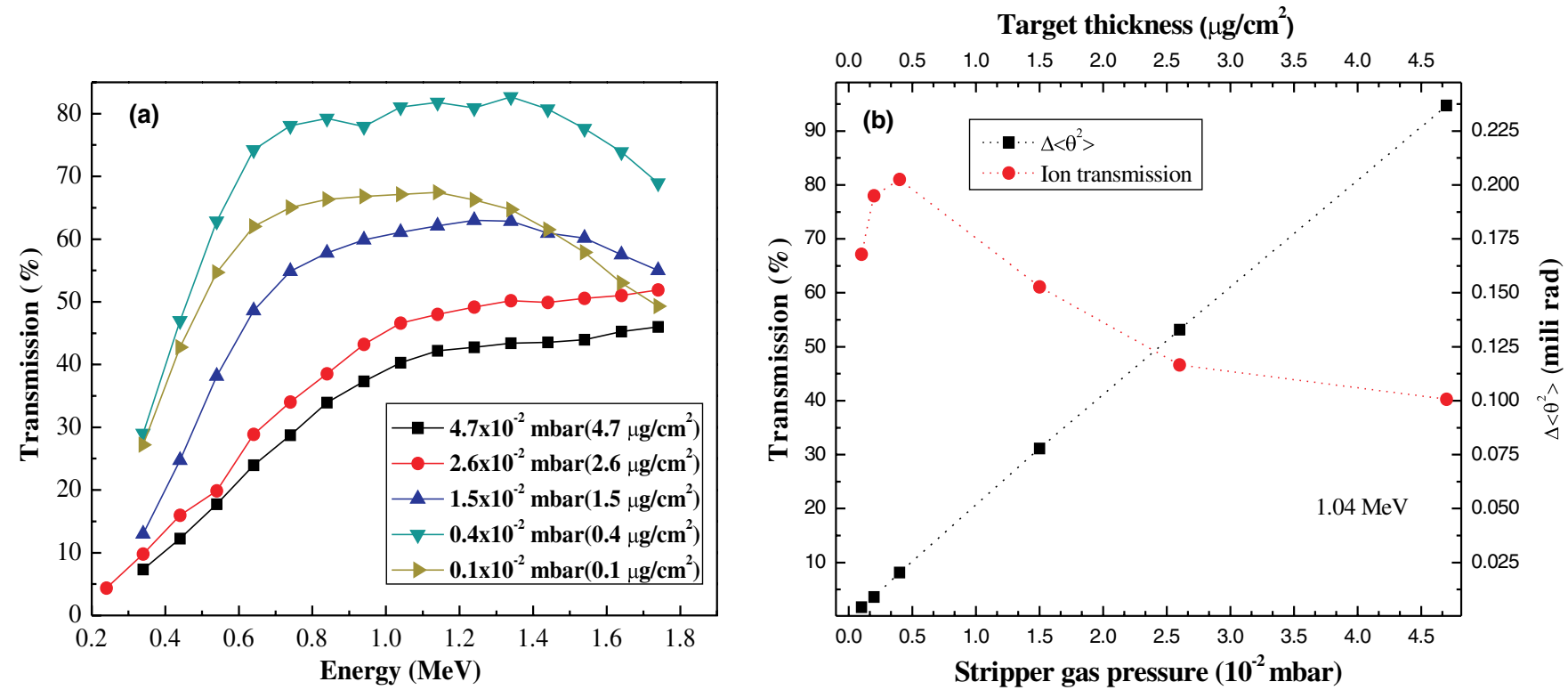

FIG. 4. (a) Variations of transmissions of the incident ${ }^{12} \mathrm{C}^{1-}$ ions with incident ion energy at different stripper gas pressures. (b) Variation of transmission, and change in mean square scattering angle $\left(\Delta\left\langle\theta^{2}\right\rangle\right)$ with stripper gas pressure at $1.04 \mathrm{MeV}$. 
dominates in the transmission behavior. The maximum ion transmission occurs at $0.4 \times 10^{-2}$ mbar, and Fig. 3 shows that the target thickness for this stripper canal pressure belongs to the nonequilibrium region of the target thicknesses. With further increment in stripper canal pressure $\left(1.5-4.7 \times 10^{-2}\right.$ mbar) the transmission $(N)$ decreases due to dominating multiple scattering inside the stripper canal. Our estimations of $\Delta\left\langle\theta^{2}\right\rangle$ at $1.5 \times 10^{-2}$ mbar and $4.7 \times 10^{-2} \mathrm{mbar}$ are 0.08 and $0.24 \mathrm{mrad}$, respectively. The above pressures are in the equilibrium target thickness range and transmission reduces by $34 \%$ in this range as apparent in Fig. 4(b). At a stripper gas pressure of $4.7 \times 10^{-2}$ mbar, the transmission is reduced by almost $50 \%$ of that at $0.4 \times 10^{-2}$ mbar. From Figs. 2(a) and 4(b), we can see that, as the stripper canal pressure increases from $0.4 \times 10^{-2}$ to $4.7 \times 10^{-2} \mathrm{mbar}$, the average charge at $1.04 \mathrm{MeV}$ increases by only $4.8 \%$, but the reduction in overall transmission is around $50 \%$. The numbers indicate that achieving higher charge state fractions in the outgoing beam by increasing stripper gas pressure will cost transmission severely. In the energy range of $1.24-1.74 \mathrm{MeV}$, transmission decreases with energy at nonequilibrium target thicknesses. In this range due to high velocity of incident ions and low target thickness a large number of incident ${ }^{12} \mathrm{C}^{1-}$ ions escape the stripper medium without any charge changing collision and, hence, the transmission on the high energy side of the accelerator reduces.

\section{Charge state fractions and their distribution}

Here we get back to discuss the reason for the higher values of experimental average charge in comparison with the predicted value for incident ion energy below $1 \mathrm{MeV}$. The mismatch between the predicted value and the experimental value of average charge at low energies is due to increased number of neutrals at low energies which we do not take into account while calculating $q_{\mathrm{avg}}^{\exp }$. We are

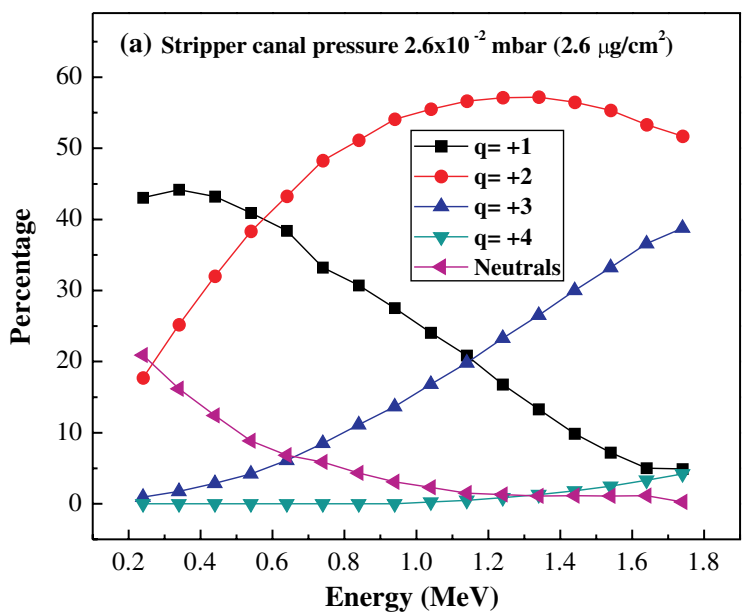

unable to measure the neutral particles $\left(N_{0}\right)$, with the experimental setup we have used for the present study. We calculate the experimental average charge $\left(q_{\text {avg }}^{\exp }\right)$ by considering only the charged carbon ions of the outgoing beam. Thus, to calculate $F_{q}$ we use

$$
F_{q}=N_{q} / \sum_{q=1}^{4} N_{q}=N_{q} / N, \quad \text { where } N=\sum_{q=1}^{4} N_{q} .
$$

Consequently, the experimental average charge value is estimated by

$$
q_{\mathrm{avg}}^{\exp }=\sum_{q=1}^{4} q F_{q}=\sum_{q=1}^{4} q\left(N_{q} / N\right) .
$$

But for low incident ion energy there will also be sufficient neutral carbon atoms in the outgoing beam and one should take them into account while estimating the fraction of different charge states and the average charge. We equate the Schiwietz's empirical value of average equilibrium charge $\left(q_{\mathrm{avg}}^{\mathrm{emp}}\right)$ with the average charge formula which includes neutrals as well, i.e.,

$$
q_{\mathrm{avg}}^{\mathrm{emp}}=\sum_{q=0}^{4} q F_{q}=\sum_{q=1}^{4} q\left[N_{q} /\left(N+N_{0}\right)\right],
$$

where $N_{0}$ is the normalized number of neutral particles in the outgoing beam. Using Eqs. (4) and (5) we can easily estimate $N_{0}$ and it can be shown that $N_{0}=N \times$ $\left(q_{\mathrm{avg}}^{\exp } / q_{\mathrm{avg}}^{\mathrm{emp}}-1\right)$. Once the normalized numbers of neutral $\left(N_{0}\right)$ and other charge particles are obtained, it is straightforward to calculate their fraction in the beam using the formula $F_{q}=N_{q} / \sum_{q=0}^{4} N_{q}$. As the empirical model predicts the equilibrium average charge, therefore to achieve good estimation of neutrals with the procedure mentioned above, it is important to use the experimental data which

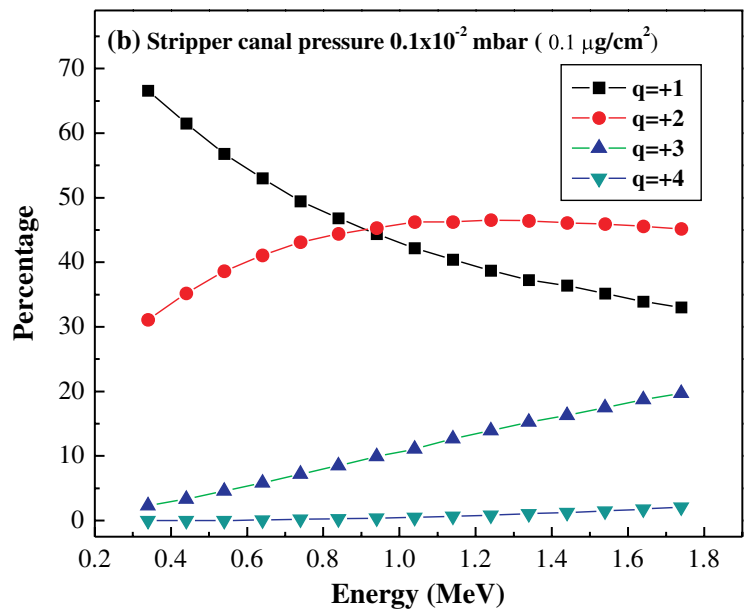

FIG. 5. (a) Variation of percentage of charge states fractions with the incident ion energy at the stripper gas pressure of $2.6 \times 10^{-2}$ mbar. The neutrals were estimated from the mismatch between the experimental and the empirical value of the average charge. (b) Variation of percentage of charge state fractions at $0.1 \times 10^{-2}$ mbar stripper gas pressure. 

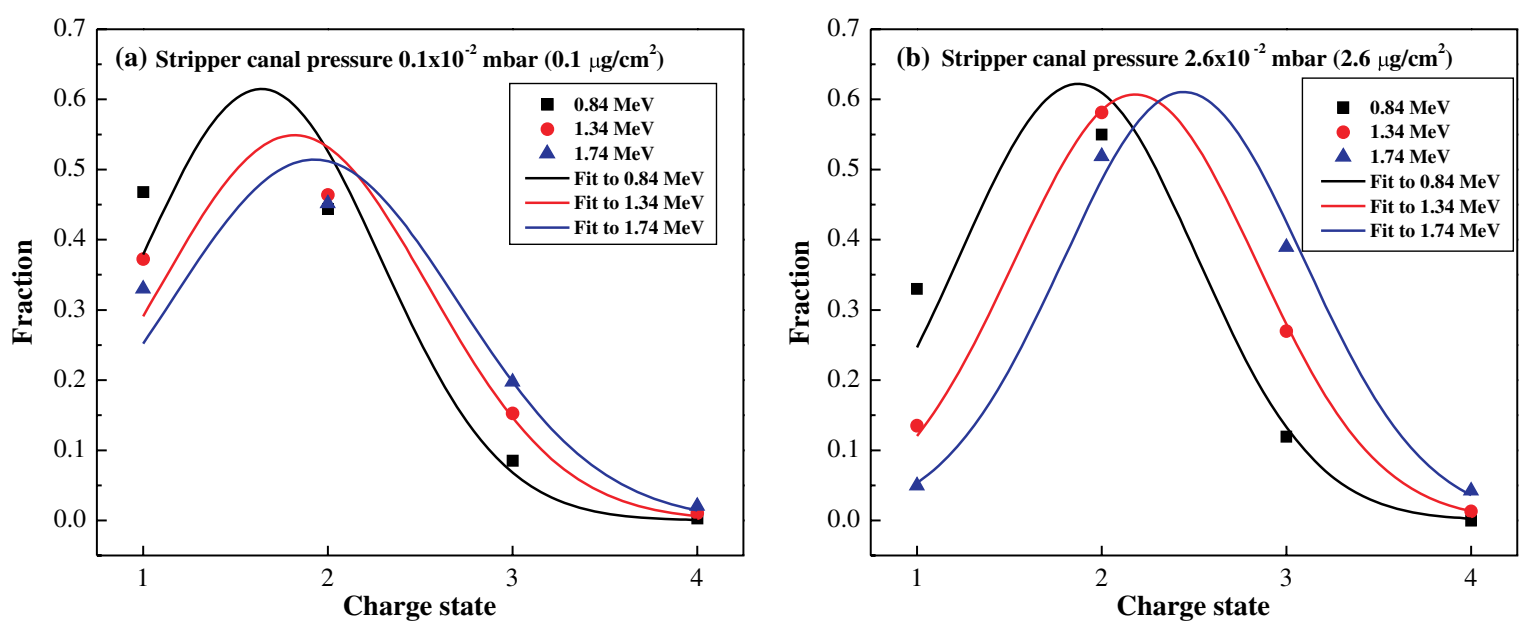

FIG. 6. Fractional distribution of charge states at different incident ion energies measured at stripper gas pressure (a) $0.1 \times 10^{-2}$ mbar and (b) $2.6 \times 10^{-2}$ mbar.

belongs to the range corresponding to equilibrium target thickness. Keeping this in mind we estimated fractions of neutrals and other charge states at stripper gas pressure $2.6 \times 10^{-2}$ mbar and their variation with energy is shown in Fig. 5(a). The percentage of neutrals goes down to less than $7 \%$ for incident ion energy $0.8 \mathrm{MeV}$. The fraction of ${ }^{12} \mathrm{C}^{2+}$ dominates in the outgoing beam in the energy range $0.5-1.7 \mathrm{MeV}$.

We have fitted Gaussians centered at average charges to the experimentally obtained charge state fractions with widths $(d)$ calculated by the definition given in the earlier section of the manuscript. Figure 6 shows the Gaussian fits to the charge state distributions at $0.84,1.24$, and $1.74 \mathrm{MeV}$ incident ion energies for stripper gas pressures $0.1 \times 10^{-2}$ and $2.6 \times 10^{-2}$ mbar, respectively. The Gaussian fits to the charge state distributions at stripper gas pressure $0.1 \times 10^{-2}$ mbar which belongs to nonequilibrium are all skewed whereas the Gaussian fits at $2.6 \times 10^{-2} \mathrm{mbar}$ belonging to equilibrium represents the distributions well. The skewness $s=\sum_{q}\left(q-q_{\text {avg }}\right)^{3} / d^{3}$ represents the asymmetry in the distribution. Figure 7 show the variation of experimental values of $s$ and $d$ with the incident ion energy in the range $0.64-1.74 \mathrm{MeV}$ at different stripper gas pressures. For equilibrium stripper gas pressures of $2.6 \times 10^{-2}$ and $4.7 \times 10^{-2}$ mbar, skewness attains a minimum at $1.24 \mathrm{MeV}$ of energy. Figure 5(a) shows that at $2.6 \times$ $10^{-2}$ mbar pressure for $1.24 \mathrm{MeV}$ incident energy the charge state fractions for $q=+1$ and $q=+3$ become almost equal while the fraction of $q=+4$ is almost zero; this results in a minima in skewness with $q_{\text {avg }}=$ 2.1. With further increase in energy, the fraction for $q=+3$ increases and $q=+1$ decreases, resulting in an increment in skewness. For a nonequilibrium value of stripper gas pressure (e.g. $0.1 \times 10^{-2}$ mbar), the skewness
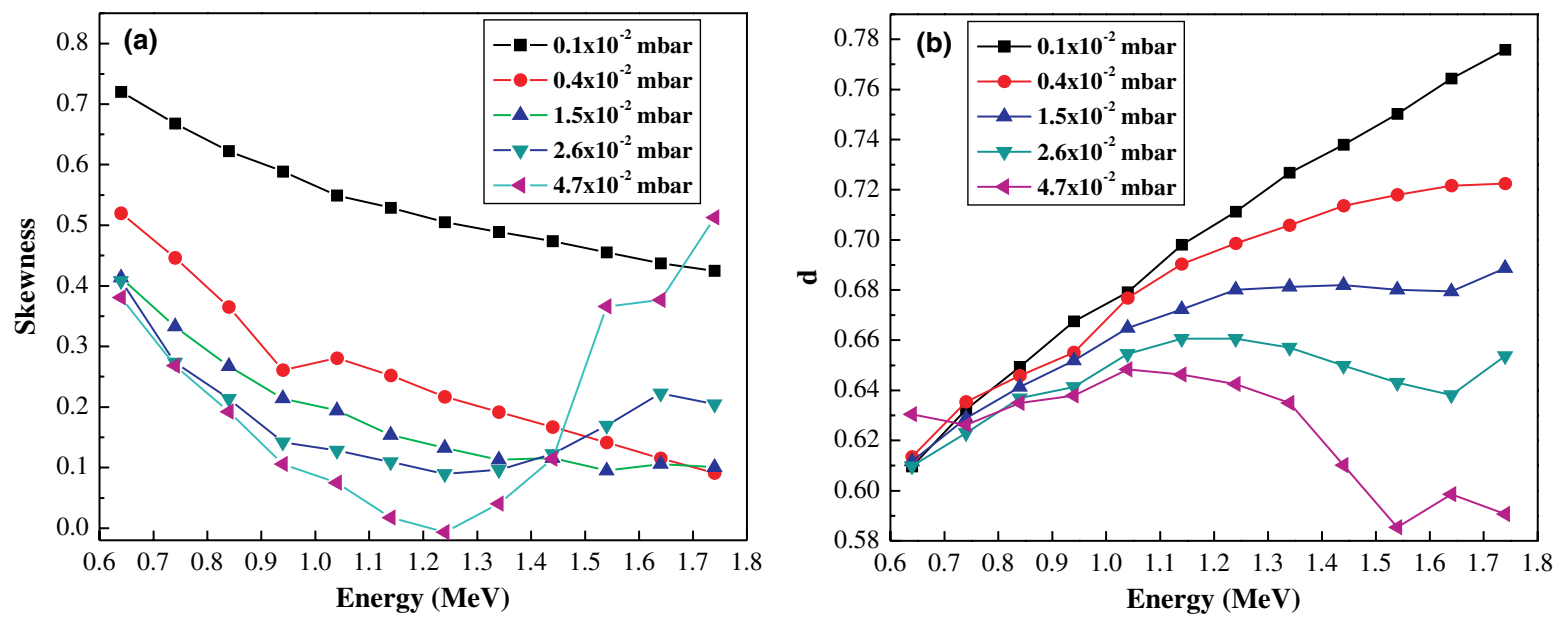

FIG. 7. (a) Variation of skewness of the charge state distribution with the incident ion energy at different stripper gas pressures. (b) Variation of the width of charge state distribution with the incident ion energy at different stripper gas pressures. 
is high for the entire range of incident ion energy, and the reason can be readily understood by looking at the charge state fractions shown in Fig. 5(b). The width of the distribution $(d)$ was approximated semiempirically by Dmitriev and Nikolaev [12]. They derived that $d=d_{1} Z^{w}$, where $d_{1}$ and $w$ were semiempirically found parameters, having values 0.32 and 0.45 , respectively, in $\mathrm{N}_{2}$ stripper gas, suggesting $d=0.71$ in the present study. The widths of the distribution are going beyond this limit for nonequilibrium stripper gas pressures, which is apparent in Fig. 7(b).

\section{CONCLUSIONS}

In this work, we have summarized experimental results of the effect of stripper gas pressure on average charge $\left(q_{\text {avg }}\right)$, charge state fractions $\left(F_{q}\right)$, and transmission $(N)$ of incident ${ }^{12} \mathrm{C}^{1-}$ ions stripped in $\mathrm{N}_{2}$ in a tandem accelerator in the energy range $0.2-1.7 \mathrm{MeV}$. The average charge data is compared with an empirical model for equilibrium average charge and good agreement is observed for equilibrium target thicknesses in the high energy side of the incident ion energy range. Stripper gas pressure has shown a small but noticeable effect in the average charge in equilibrium range. The transmission study shows that the maximum transmission of incident ions is achieved at a nonequilibrium thickness of the target medium. The study of charge state distributions together with the transmission is valuable for optimized use of a tandem accelerator for high current applications.

\section{ACKNOWLEDGMENTS}

We thank late Professor V.N. Kulkarni, Professor H. C. Verma, and Professor Sudeep Bhattacharjee for fruitful discussions and useful suggestions. We acknowledge the students and staff members of ion beam laboratory, IIT Kanpur, for their help during the experiments. Financial support from the Department of Science and Technology, Government of India is gratefully acknowledged.

[1] M. C. Ridgway, G. de M. Azevedo, R. G. Elliman, C. J. Glover, D. J. Llewellyn, R. Miller, W. Wesch, G. J. Foran, J. Hansen, and A. Nylandsted-Larsen, Phys. Rev. B 71, 094107 (2005).

[2] S. Budak, S. Guner, R. A. Minamisawa, and D. ILA, Surf. Coat. Technol. 203, 2479 (2009).

[3] N. Bohr, K. Dan. Vidensk. Selsk. Mat. Fys. Medd. 18, 1 (1948).

[4] N. Bohr and J. Lindhard, K. Dan. Vidensk. Selsk. Mat. Fys. Medd. 28, 1 (1954).

[5] H. D. Betz, IEEE Trans. Nucl. Sci. 18, 1110 (1971).

[6] C. Schmitt, J. A. LaVerne, D. Robertson, M. Bowers, W. Lu, and P. Collon, Nucl. Instrum. Methods Phys. Res., Sect. B 268, 1551 (2010).

[7] C. Schmitt, J. A. LaVerne, D. Robertson, M. Bowers, W. Lu, and P. Collon, Phys. Rev. A 80, 052711 (2009).

[8] M. Kiisk, B. Erlandsson, M. Faarinen, R. Hellborg, K. Håkansson, P. Persson, G. Skog, and K. Stenström, Nucl. Instrum. Methods Phys. Res., Sect. A 481, 1 (2002).

[9] M. Kiisk, R. Hellborg, P. Persson, M. Faarinen, G. Skog, and K. Stenström, Nucl. Instrum. Methods Phys. Res., Sect. A 521, 299 (2004).

[10] S. A. W. Jacob, M. Suter, and H.-A. Synal, Nucl. Instrum. Methods Phys. Res., Sect. B 172, 235 (2000).

[11] G. Schiwietz and P. L. Grande, Nucl. Instrum. Methods Phys. Res., Sect. B 175-177, 125 (2001).

[12] H. D. Betz, Rev. Mod. Phys. 44, 465 (1972).

[13] D. Dinev, Phys. Part. Nucl. 40, 257 (2009).

[14] B. Franzke, in CAS - 4th Advanced Accelerator Physics Course, Noordwijkerhout, 1991, edited by S. Turner (CERN 1992-01), p. 100.

[15] G. Ryding, A. Wittkower, and P. H. Rose, Phys. Rev. 184, 93 (1969). 